\title{
Developing New Methods for the Mono- end Functionalization of Living Ring Opening Metathesis Polymers
}

\author{
Andreas F. M. Kilbinger*
}

\begin{abstract}
In this article we present a review of our recent results in one area of research we are involved in. All research efforts in our group focus on functional polymers and new ways of gaining higher levels of control with regard to the placement of functional groups within these polymers. Here, the living ring opening metathesis polymerization (ROMP) will be reviewed for which end-functionalization methods had been rare until very recently. Polymers carrying particular functional groups only at the chain-ends are, however, very interesting for a variety of industrial and academic applications. Polymeric surfactants and polymer-protein conjugates are two examples for the former and polymer- $\beta$-sheet-peptide conjugates one example for the latter. The functionalization of macroscopic or nanoscopic surfaces often relies on mono-end functional polymers. Complex macromolecular architectures are often constructed from macromolecules carrying exactly one functional group at their chainend. The ring opening metathesis polymerization is particularly interesting in this context as it is one of the most functional group tolerant polymerization methods known. Additionally, high molecular weight polymers are readily accessible with this technique, a feature that living radical polymerizations often struggle to achieve. Finding new ways of functionalizing the polymer chain-end of ROMP polymers has therefore been a task long overdue. Here, we present our contribution to this area of research.
\end{abstract}

Keywords: Ring opening metathesis polymerization (ROMP)

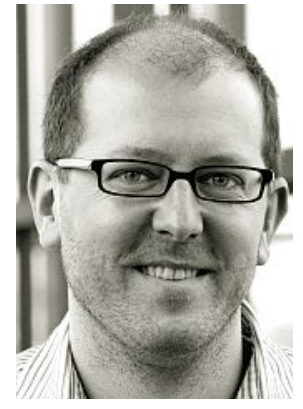

Andreas Kilbinger studied chemistry at the Universities of Mainz and Berlin (Freie Universität) where he received his diploma in chemistry in 1996. After that he moved to the University of Durham
(United Kingdom) where he obtained his $\mathrm{PhD}$ in polymer chemistry at the IRC in Polymer Science and Technology under the supervision of Prof. W. J. Feast. This was followed by two years of postdoctoral studies at the California Institute of Technology (Caltech) in the research group of Prof. R. H. Grubbs funded via a Feodor Lynen Fellowship by the Alexander von Humboldt Foundation. He then returned to the University of Mainz to the group of Prof. H. Frey where he received his habilitation in organic chemistry in 2007. The same year he received the Hermann Schnell-Prize awarded by the German Chemical Society. He joined the chemistry department of the University of Freiburg in November 2010 as a professor for polymer chemistry. His group focuses on the end-functionalization of living olefin metathesis polymers and the synthesis and self-assembly of shape-persistent supramolecular polymers.

\section{Introduction}

Olefin metathesis polymerization has been known for many years. In the early days ill-defined initiators were used for polymerization of cyclic olefins. The first well-defined polymerization initiators were based on titanium complexes. ${ }^{[1]}$ Later on, well-defined metal carbene complexes based on molybdenum and tungsten ${ }^{[2]}(3$ and 4 in Fig. 1) were developed by the Schrock group. Olefin metathesis active ruthenium carbene complexes ( $\mathbf{1}$ and $\mathbf{2}$ in Fig. 1) were developed by Grubbs et al. Many of these well-defined metal-carbene complexes allow the synthesis of so-called living polymers. Here, a fast initiating complex consumes monomer in the absence of significant side-reactions such as unwanted chain transfer or termination reactions.

A schematic representation of the ring opening metathesis polymerization us-

\footnotetext{
${ }^{*}$ Correspondence: Prof. Dr. A. Kilbinger

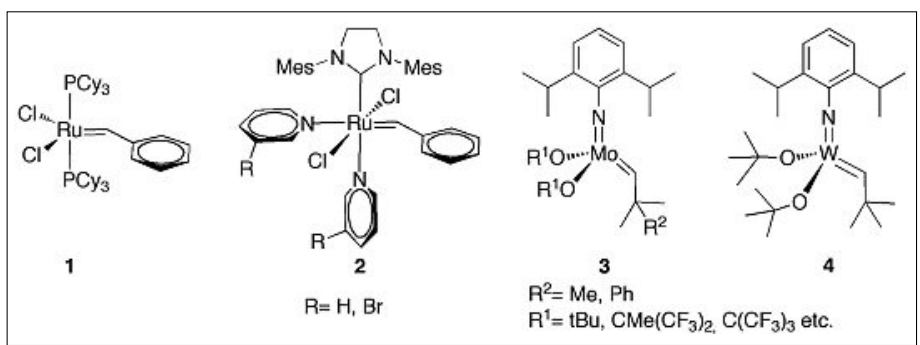

Fig. 1. The most common metal-carbene complexes used for ring opening metathesis polymerization. 


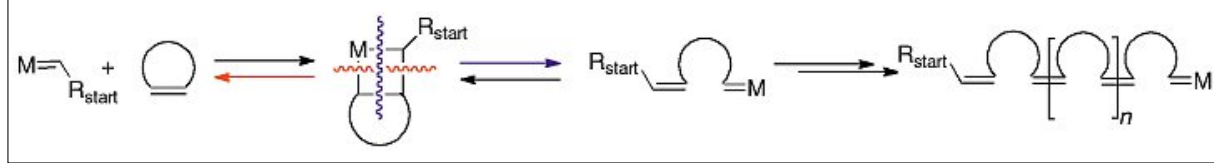

Scheme 1. Ring opening metathesis polymerization. Red and blue wavy lines: possible cleavage pathways of the metallacyclobutane.

Table 1. The functional group tolerance of transition metal carbene complexes

\begin{tabular}{|c|c|c|c|}
\hline Titanium & Tungsten & Molybdenum & Ruthenium \\
\hline Carboxylic acids & Carboxylic acids & Carboxylic acids & Olefins \\
\hline Alcohols, Water & Alcohols, Water & Alcohols, Water & Carboxylic acids \\
\hline Aldehydes & Aldehydes & Aldehydes & Alcohols, Water \\
\hline Ketones & Ketones & Olefins & Aldehydes \\
\hline Esters, Amides & Olefins & Ketones & Ketones \\
\hline Olefins & Esters, Amides & Esters, Amides & Esters, Amides \\
\hline
\end{tabular}

ing metal-carbene initiators is shown in Scheme 1. The metal-carbene complex $[\mathrm{M}]=\mathrm{CHR}_{\text {start }}$ reacts with a strained cyclic olefin to form a metallacyclobutane intermediate which can in principle cleave in two ways, depicted in red and in blue. The red pathway illustrates the back reaction whereas the blue pathway leads to the ring opening of the strained cyclic olefin and the formation of a new metal-carbene complex. This newly formed complex can continue to propagate, i.e. open strained cyclic olefins until all substrate is consumed.

Nonetheless, the different metal-carbene complexes available today differ tremendously not only in their reactivity but also in their functional group tolerance. A look at Table 1 shows that the early transition metal carbene complexes which are more oxophilic react readily with oxygen containing functional groups. In titanium reagents such as the Tebbe complex ${ }^{[3]}$ or in the McMurry reaction ${ }^{[4]}$ it is this oxophilicity that is exploited. Tungsten carbene complexes tolerate esters and amides to be present during olefin metathesis and molybdenum carbene complexes additionally tolerate ketones. Both, however, react with aldehydes in Wittig-like reactions, transferring the aldehyde residue onto the chain-end of the polymer thus allowing for controlled mono end-functionalization. ${ }^{[5-9]}$

In other words, Table 1 shows some functionalities listed below Olefins which are tolerated by the carbene complex and others above Olefins which might be used for a selective termination/polymer functionalization reaction. Ruthenium carbene complexes are the most functional group tolerant metathesis active complexes and have therefore been used extensively in organic chemistry small molecule transformations.

Functional group tolerance is a great feature for ring closing and cross metathesis applications and also for the synthesis of highly functional polymers. However, which reagents can we use with propagating ruthenium carbene complexes in order to selectively turn them into a defined polymer end-group? This general problem is illustrated in Fig. 2 where the living ring opening metathesis polymerization is compared to living anionic polymerization. In the latter a carbanion is the propagating species which has a high reactivity and in turn does not tolerate the presence of many electrophilic functional groups within the monomer structure. This limits the types of polymers that are accessible via carbanionic polymerization but also allows us to choose from a relatively large pool of re-

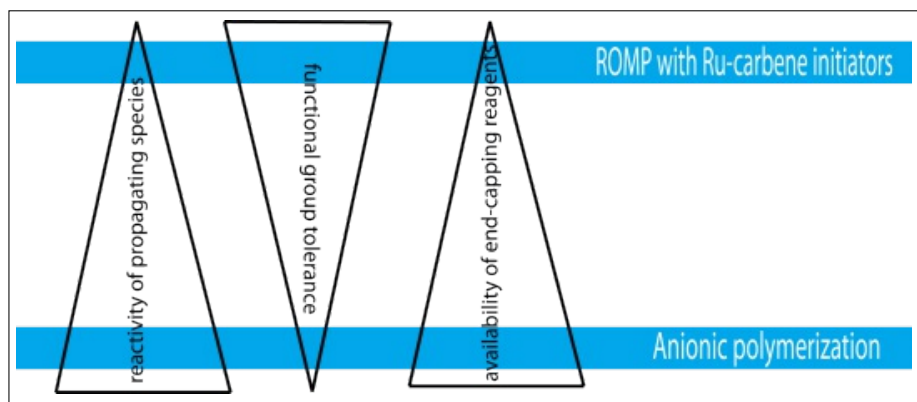

Fig. 2. A schematic comparison of living ring opening metathesis polymerization with ruthenium carbene complexes as the propagating species and living anionic polymerization. agents that react cleanly with the carbanion in a terminal functionalization reaction.

Ruthenium carbene complexes on the other hand tolerate many functional groups, i.e. they show low or no reactivity towards them. All of these functionalities can thus be present during polymerization (for example as part of the monomer structure) but consequently none of these can be used for functional termination.

The focus of our research activity reviewed here is to find new ways of turning the living ruthenium carbene complex at the chain-end of a polymer into a variety of functional groups.

\section{General Methods of End- functionalization}

The area of living ROMP ${ }^{[10]}$ as well as the end-functionalization of ROMP[11] has recently been comprehensively reviewed. Here we will report mainly on the research that has been carried out in our group. Table 1 shows clearly that ruthenium carbene complexes prefer to react with olefins over many other functional groups. Fraser et al. had polymerized monomers (cyclic olefins) and cyclic cleavable olefins in a statistical manner. ${ }^{[12]}$ This yielded polymers with cleavable sites scattered along the polymer chain. Subsequent cleavage of these sites gave so-called telechelic polymers, i.e. polymers with functional groups at either polymer chain-end. Due to the statistical incorporation of the cleavable monomer into the polymer chain the telechelic polymers produced showed a broader molecular weight distribution than typically achieved for living polymerizations.

In our very first approach to introduce polymer end-groups via ROMP we used the cleavable cyclic monomers described by Fraser et al. in a block copolymer synthesis (Fig. 3, left). ${ }^{[13,14]}$ The first polymer block was prepared from a norbornene derivative (exo- $N$-phenylnorbornene-2,3-dicarboximide) and the second block from a substituted dioxepine (isopropyl dioxepine or phenyl dioxepine). The second polyacetale block could be cleaved under acidic conditions after polymerization. This resulted in exactly one allylic alcohol group covalently attached to the first polymer block. Successful synthesis could also be followed by gel permeation chromatography (GPC). The GPC traces shown in Fig. 3 (right) show the decrease of molecular weight when the second block in the diblock copolymer is 'sacrificed'/degraded.

The same strategy could also be exploited for the synthesis of allylic thiol end-groups. ${ }^{[15]}$ A phenyl dithiepine was used as the sacrificial monomer for the synthesis of the second polymer block. The polydithiepine block could be 'sacrificed' 

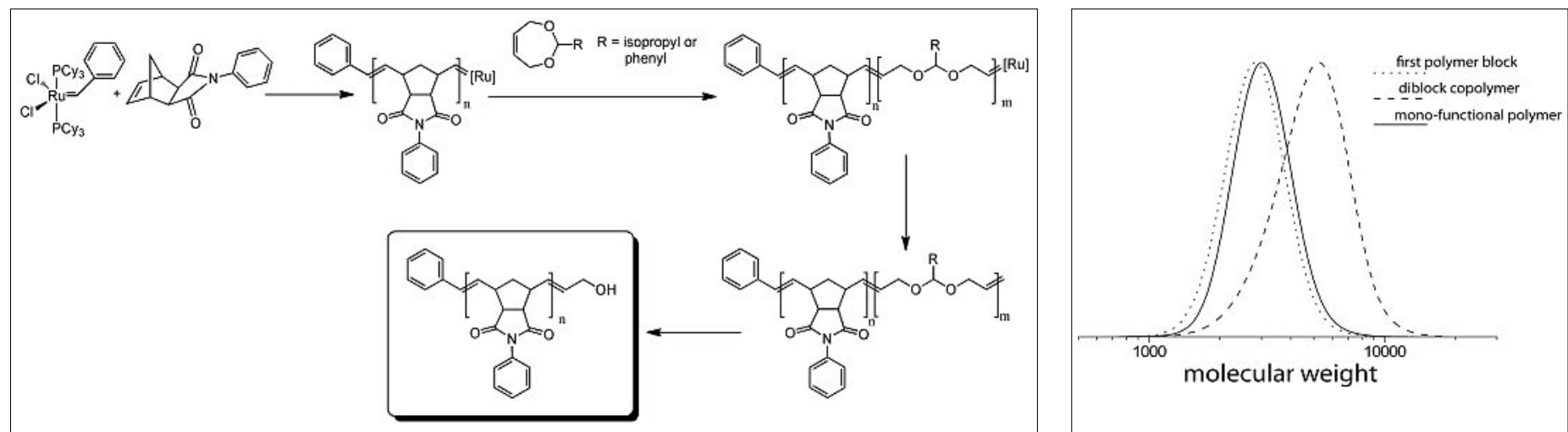

Fig. 3. Left: Polymer carrying terminal allylic alcohols via sacrificial synthesis. Right: Gel permeation chromatogram of the first polymer block (dotted line), the diblock copolymer (dashed line) and the alcohol end-functionalized polymer (solid line).

using $\mathrm{H}_{2}$ /Raney-nickel leading to thiol end-functionalized polymers that have attracted some attention in recent years for the functionalization of macroscopic and nanoscopic gold surfaces.

The allylic alcohol functionalized polymers prepared via sacrificial synthesis were also used to prepare more complex macromolecular architectures. Scheme 2 (top) shows the synthesis of a diblock copolymer consisting of a ring opening metathesis block and a poly(ethylene glycol) block. To achieve the coupling of the two blocks, the alcohol end-group was esterified with propargylic acid and subsequently reacted with an azide end-functionalized poly(ethylene glycol) in a $\mathrm{Cu}$-catalyzed 1,3-dipolar cycloaddition. ${ }^{[16]}$ This strategy allows the synthesis of diblock copolymers in which one block is prepared via ROMP and the other via a different polymerization technique.

Scheme 2 (bottom) shows a diblock copolymer with an allylic alcohol end-group prepared via the sacrificial synthesis route described above. Reaction with a norbornene carbonyl chloride gave a macromonomer which could again be polymerized via ROMP. The resulting polymer represents a graft copolymer in which the grafts themselves are diblock copolymers. ROMP was the only polymerization technique required for the synthesis of this macromolecular architecture. ${ }^{[17]}$

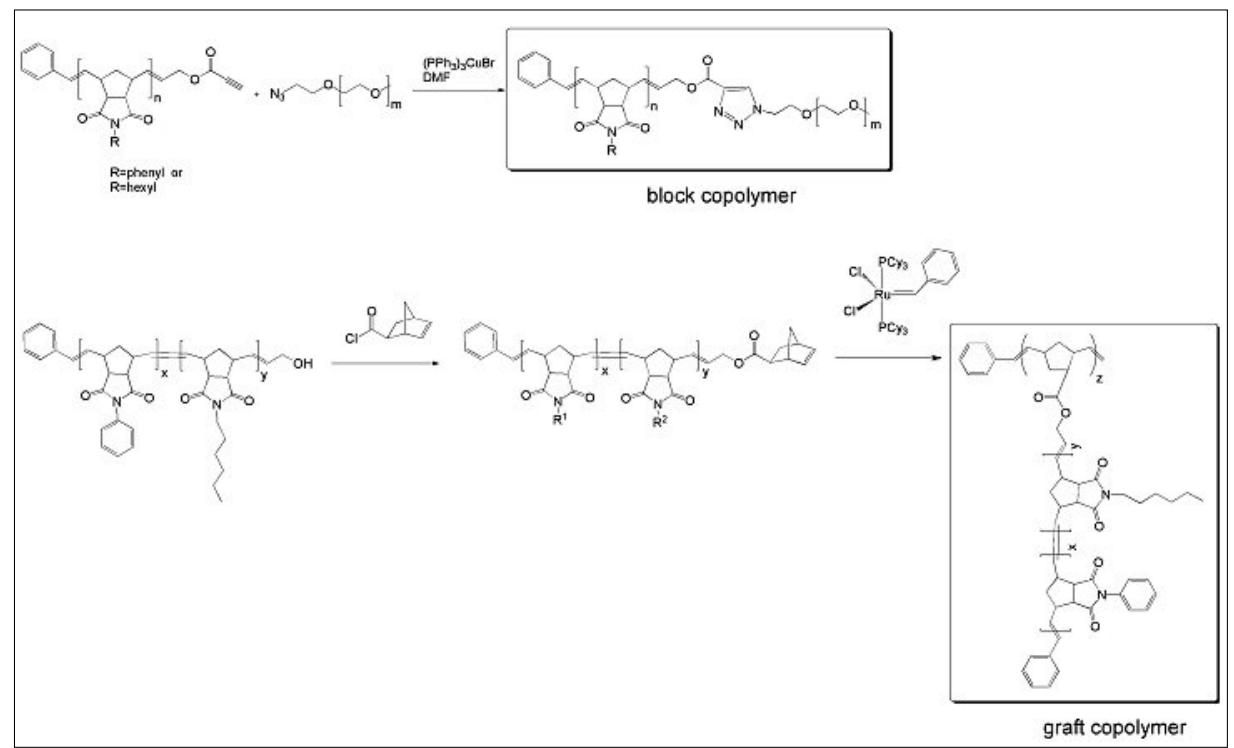

Scheme 2. Using alcohol end-functional polymers for the construction of macromolecular architectures. Top: synthesis of a diblock copolymer. Bottom: Synthesis of a graft copolymer in which the graft itself represents a diblock copolymer.

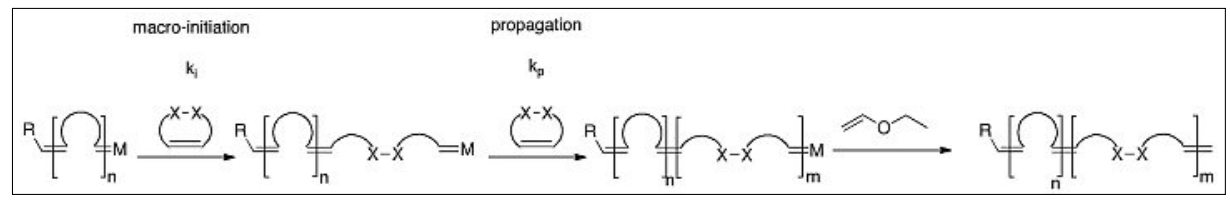

Scheme 3. Representation of the key steps involved in sacrificial synthesis that determine the degree of end-group functionalization.
In order to achieve high degrees of endgroup functionality using the sacrificial synthesis route, every single propagating chain-end of the first polymer block has to react with a sacrificial monomer. The degree of macroinitiation is therefore important for high degrees of end-functionality. The propagation of the sacrificial monomer merely consumes sacrificial monomer without leading to higher degrees of functionalization after cleavage of the sacrificial block (Scheme 3 ). In order to quantify the amounts of sacrificial monomer needed to obtain high degrees of end-functionality, we used an equation derived by Szwarc for living anionic polymerization. ${ }^{[18-20]}$

According to this equation (Eqn. (1)) the initiation efficiency of an initiator can be calculated from the $\mathrm{k}_{\mathrm{p}} / \mathrm{k}_{\mathrm{i}}$ factor (= ratio of the rate constants for propagation and initiation) and the number of equivalents of monomer added to the initiator. In the case of sacrificial synthesis, $M_{\text {total }} / \mathrm{C}_{\text {total }}$ is defined by the number of equivalents of sacrificial monomer per initiator $\left(\mathrm{M}_{\text {total }}\right.$ : monomer concentration, $\mathrm{C}_{\text {total }}$ : initiator concentration) and the initiation efficiency $f$ represents the degree of end-functionalization. As ROMP represents a reversible reaction, the values for $\mathrm{k}_{\mathrm{p}}$ and $\mathrm{k}_{\mathrm{i}}$ are apparent values.

$$
\frac{M_{\text {total }}}{C_{\text {total }}}=\left(\frac{k_{p}}{k_{i}}\right) \cdot\left[\ln (1-f)^{-1}-f\right]+f
$$

Determining the degree of end-functionality for different ratios of sacrificial monomer to macroinitiator allowed us to fit Eqn. (1) and to obtain a value for $\mathrm{k}_{\mathrm{p}} / \mathrm{k}_{\mathrm{i}}$ which is unique for each sacrificial monomer. These values allow the calculation of the minimum amounts of each sacrificial monomer necessary to yield high degrees of end-functionalization.

Sacrificial synthesis is a great way of end-functionalizing ROMP polymers. It is easy to carry out and successful endfunctionalization can in principle be verified with relatively low analytical effort 


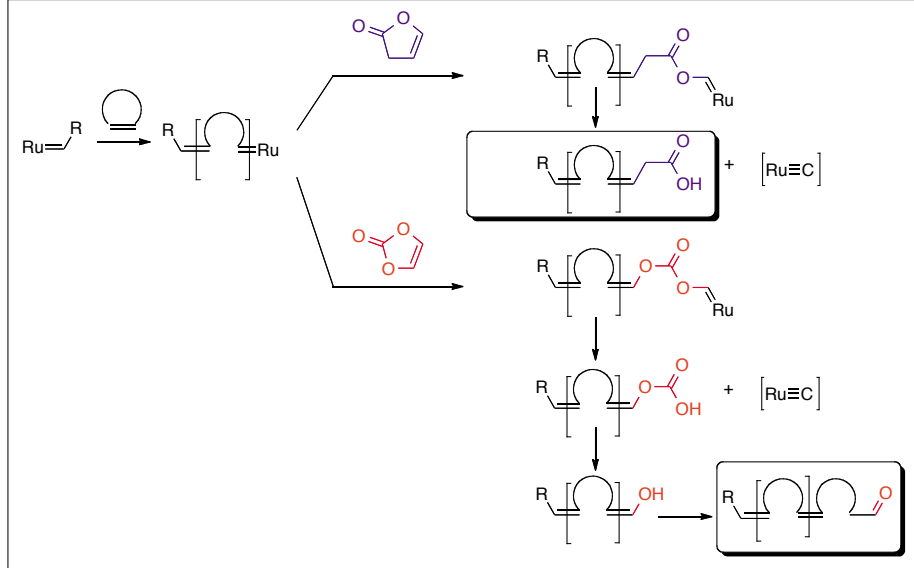

Scheme 4. ROMP end-functionalization using $3 \mathrm{H}$-furanone and vinylene carbonate. for example size exclusion chromatograms before and after cleavage of the sacrificial block. However, it is a two-step process, requires liberation of the macroscopically protected end-functionality and is certainly not 'atom-economic'.

Vinyl lactones can also be used for endfunctionalization of living ROMP polymers. Scheme 4 shows the two examples reported to date that yield polymers bearing terminal carboxylic acids or aldehydes. ${ }^{[21]}$ As shown in the scheme, $3 H$-furanone (blue top pathway) reacts with the propagating ruthenium carbene end-group to give an acyl carbene which decomposes into a carbido complex and a polymer terminal carboxylic acid. The carbido complex is metathesis inactive and no longer covalently attached to the polymer chain. A simple precipitation of the crude polymer mixture therefore yields the monoend-functional polymer. $3 H$-furanone can readily be prepared from furfural via a Baeyer-Villiger oxidation.

Using the commercially available vin- ylene carbonate (red bottom pathway in the scheme) a similar acyl carbene complex is formed which also yields the ruthenium carbido complex. After decarboxylation a vinyl alcohol is formed at the polymer chain-end which tautomerizes into the terminal aldehyde.

With a combination of the two methods, sacrificial synthesis and vinyl lactone termination, we can prepare living ROMP polymers that carry two different functional groups at either end of the polymer chain (Scheme 5). We first initiate the polymerization of methyl dioxepine as the first sacrificial block. Methyl dioxepine was chosen as it is the most volatile polymerizable dioxepine and therefore allows for traces to be removed by vacuum before introducing the monomer for the second 'permanent' polymer block. After the polymerization of the second block is complete the propagating ruthenium carbene chainend is functionally terminated with either $3 H$-furanone or vinylene carbonate. This end-capping procedure yields carboxylic

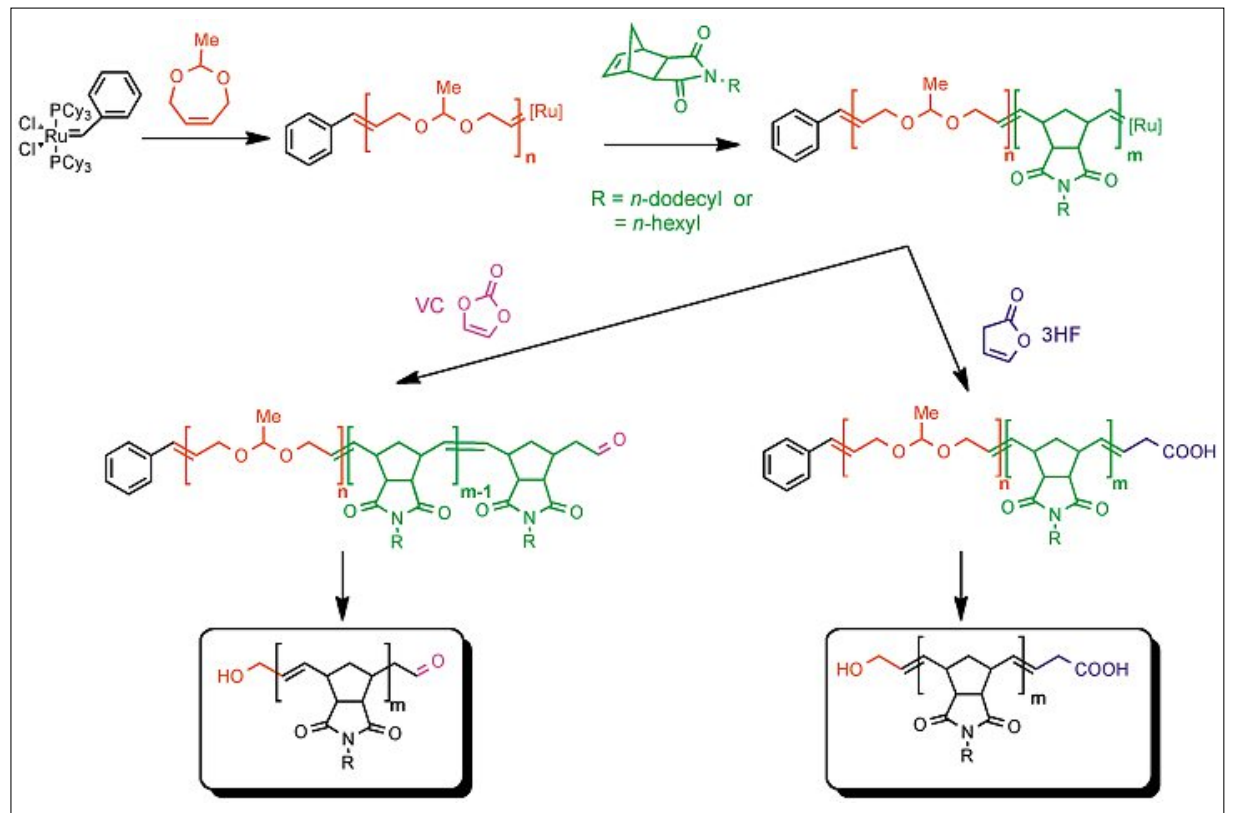

Scheme 5. Heterotelechelic ROMP polymers carrying one allylic alcohol group at the start of the polymer chain and either a carboxylic acid or an aldehyde group at the chain-end. acid or aldehyde end-groups. After that, the first sacrificial block is cleaved giving one single allylic alcohol function at the start of the polymer chain. The combination of these two methods therefore yields so-called heterotelechelic polymers which carry two different functional end-groups.

\section{Conclusions and Outlook}

This personal review has focused only on the work going on in our research group in Freiburg/Mainz. Older end-group functionalization methods that are still being used today such as substituted vinyl ethers have not been addressed at all in this article. Since we entered the field of ROMP end-functionalization, Grubbs et al. have shown that terminal cross-metathesis is another very effective and straight forward way of end-functionalizing polymers. This method has also not been reviewed here. Two recent comprehensive reviews of the field summarize all different end-functionalization methods available today. ${ }^{[11]}$

What remains to be done in this area of research? We are currently working on prefunctionalizable end-capping substrates that selectively terminate the ROMP polymerization and transfer the pre-functionalized group onto the chain-end of the polymer. Such a 'universal end-capping reagent' would be atom economical, showing fast end-capping kinetics and could be derivatized with a large variety of different groups and/or functionalities.

Another area that has been somewhat neglected is the use of pre-functionalized initiators. If the initiator carries the correct (possibly protected) functional group then all polymer chains formed must by definition be correctly functionalized. Functional initiators therefore have the potential of yielding very high degrees of chain-end (better chain-start) functionality.

Both areas are currently being addressed by our group and we will report first results in due course.

\section{Acknowledgements}

AFMK thanks Mark Schäfer and Nils Hanik for valuable discussions.

Received: January 30, 2012

[1] N. Calderon, E. A. Ofstead, J. P. Ward, W. A. Judy, K. W. Scott, J. Am. Chem. Soc. 1968, 90, 4133.

[2] R. R. Schrock, Angew. Chem. Int. Ed. 2006, 45, 3748.

[3] F. N. Tebbe, G. W. Parshall, G. S. Reddy, J. Am. Chem. Soc. 1978, 100, 3611.

[4] J. E. McMurry, M. P. Fleming, J. Am. Chem. Soc. 1974, 96, 4708.

[5] R. R. Schrock, Acc. Chem. Res. 1990, 23, 158.

[6] R. R. Schrock, J. Feldman, L. F. Cannizzo, R. H. Grubbs, Macromolecules 1987, 20, 1169.

[7] J. S. Murdzek, R. R. Schrock, Macromolecules 1987, 20, 2640. 
[8] R. R. Schrock, J. S. Murdzek, G. C. Bazan, J. Robbins, M. DiMare, M. B. O'Reagan, J. Am. Chem. Soc. 1990, 112, 3875.

[9] G. C. Bazan, E. Khosravi, R. R. Schrock, W J. Feast, V. C. Gibson, M. B. O'Reagan, J. K. Thomas, W. M. Davis, J. Am. Chem. Soc. 1990, 112,8378 .

[10] C. W. Bielawski, R. H. Grubbs, Prog. Polym. Sci. 2007, 32, 1 .

[11] a) K. Nomura, M. M. Abdellatif, Polymer 2010, 51,1861 ; b) S. Hilf, A. F. M. Kilbinger, Nature Chem. 2009, 1, 537.
[12] C. Fraser, M. A. Hillmyer, E. Gutierrez, R. H. Grubbs, Macromolecules 1995, 28, 7256.

[13] S. Hilf, E. Berger-Nicoletti, R. H. Grubbs, A. F. M. Kilbinger, Angew. Chem. Int. Ed. 2006, 45, 8045.

[14] S. Perrier, X. Wang, Nature 2007, 445, 271.

[15] S. Hilf, A. F. M. Kilbinger, Macromolecules 2009, 42, 4127.

[16] S. Hilf, N. Hanik, A. F. M. Kilbinger, J. Polym. Sci. A 2008, 46, 2913.

[17] S. Hilf, A. F. M. Kilbinger, Macromol. Rapid Commun. 2007, 28, 1225 .
[18] M. Szwarc, Makromol. Chem. 1960, 35, 132.

[19] M. Szwarc, M. van Beylen, D. van Hoywegen, Macromolecules 1987, 20, 445.

[20] S. Hilf, R. H. Grubbs, A. F. M. Kilbinger, Macromolecules 2008, 41, 6006.

[21] S. Hilf, R. H. Grubbs, A. F. M. Kilbinger, J. Am. Chem. Soc. 2008, 130, 11040. 